\title{
Study on Application of Refined Management Method Based on Data Analysis in Expressway Toll Management
}

\author{
Qilin Sun* \\ Road Network Monitoring and Emergency Command Center of Yunnan Provincial Department of \\ Transportation, Kunming, Yunnan, 650000, China \\ *Corresponding author
}

Keywords: Expressway management, Refined management, Data analysis

\begin{abstract}
In the new era, the management of expressway toll is facing more challenges, which requires operator to innovate in management ideas, methods and means. Refined management is an important direction of expressway toll management. Firstly, we elaborate main problems of expressway toll refined management, and put forward the objectives of expressway toll refined management. Then, we propose that data mining can be used to develop the application of expressway refined management. Finally, we put forward some countermeasures and suggestions on refined management of expressway toll, from the perspective of big data application environment, financial support, privacy protection and management and service level.
\end{abstract}

\section{Introduction}

In operation and management of expressway, toll management is very important. In the new era, the management of expressway toll is facing more challenges, which requires operator to innovate in management ideas, methods and means. Refined management will be an important direction of expressway toll management. By improving the relevant rules and regulations and work requirements, we can make the expressway toll work more standardized.

At present, China's expressway has realized comprehensive network coverage. Through the construction of network platform, we can better strengthen the information exchange and resource sharing between toll stations, so as to create conditions for the realization of large-scale benefits. At present, the charge management based on the network has formed the charge mode of collecting the entrance card and paying the exit fee [1]. However, there are still many problems, e.g. the toll operation process is imperfect, and emergency capacity of congestion is weak. Luckily, in the operation and management of expressways for many years, considerable data have been accumulated [2]. Therefore, combining with data analysis technology, making full use of vehicle traffic data, vehicle owner data, public security data, etc., it can help to build the expressway refined management system.

\section{Dilemma and Objectives of Expressway Toll Refined Management}

\subsection{Dilemma}

\subsubsection{Imperfect Toll Operation Process}

The expressway tolling operation process refers to the link from passing vehicles to the toll window to the end of payment of fees, which involves several links of vehicle information recording, reading of IC cards, and calculation of settlement. The introduction of ETC achieves the goal of fully automatic information recording, but it also needs to rely on manual semi-automation to issue access cards and charges. In addition, in order to avoid abnormal situations, the electronic monitoring system needs to be continuously improved, so it forms a comprehensive management network of computer, monitoring and human participation. Although the toll collection system has been established, unskilled manual operations occur from time to time, and errors such as ticketing errors and misplaced "free cars" still occur sometimes. This has created a certain obstacle to the 
establishment of refined expressway toll management and is worthy of consideration by expressway management departments.

It should be noted that the overall toll collection process of the expressway is not perfect, and the service level still needs to be improved, which is directly related to the insufficient proficiency of employees [3]. The goal of standardized management charging process is to use scientific and modern management methods to continuously improve management methods and management levels. Every link of the charging process needs to be precise and scientific. Only in this way can it help to establish a good window image and improve service satisfaction.

\subsubsection{Inadequate Performance Appraisal System}

The performance evaluation of expressway toll involves all aspects of management, which is of great significance to promote the management level of toll stations. In fact, performance appraisal is a kind of appraisal for employees' performance. Its purpose is to stimulate employees' work enthusiasm and create more value on the basis of ensuring the completion of basic salary. Generally speaking, the performance evaluation system of expressway toll stations in China needs to be improved continuously, the performance management effect is not good, and the implementation strength needs to be strengthened. Therefore, the performance appraisal system of expressway toll collection should be established and perfected as soon as possible, so that the development of the appraisal work can be fully guaranteed and the real value of the appraisal management can be brought into play.

Firstly, employee performance is closely related to many aspects of work, so the selected assessment indicators should be as comprehensive as possible. Secondly, in the original assessment system, some regulations not only have no corresponding value but also play an obstacle role, which makes some people slack. Thirdly, how to assess the employees and how to set the content and form of the assessment are all issues that the operator should consider.

\subsubsection{Weak Emergency Capacity of Congestion}

With the continuous improvement of China's expressway network, it has become the first choice for many people to travel. But this situation also presents a higher level challenge to the current expressway toll work. On the one hand, since there are few lanes in toll stations, congestion often occurs, especially on free holidays; on the other hand, the level of hardware facilities of toll station is not perfect, and when there are problems such as equipment failure, the management personnel will take the way of closing the lane, which will reduce the charging efficiency.

If the congestion problem caused by toll station cannot be handled and controlled in time, its impact will be severe. There are three stages in the payment operation of expressway toll station: entering transition stage, charging service stage and leaving transition stage, so the three stages of vehicle passing through the toll station are shown in Figure 1.

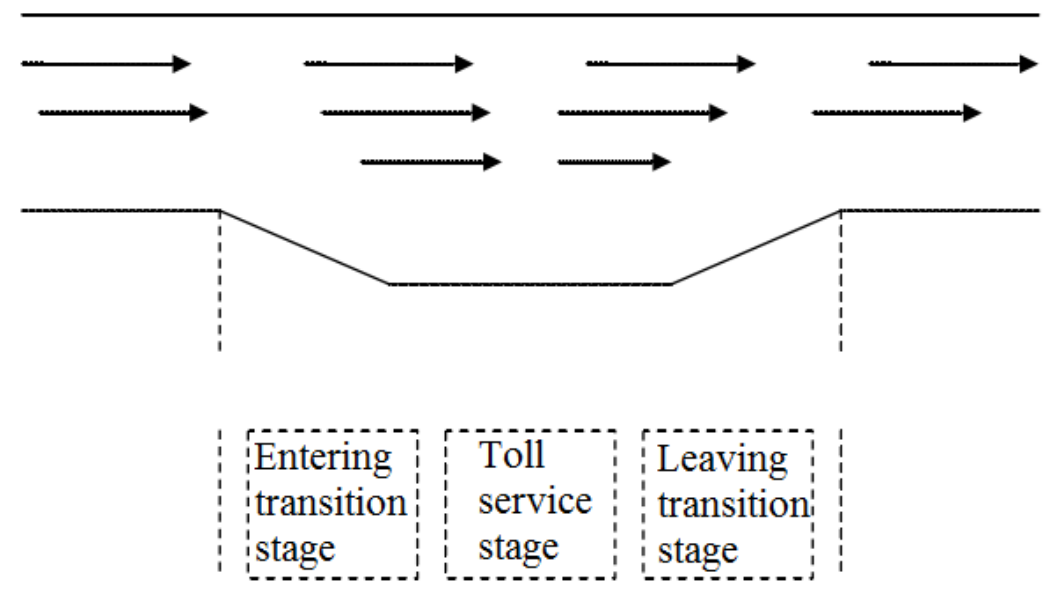

Fig. 1 Three Stages of Vehicle Passing through the Toll Station.

For expressway toll stations, they are generally located far away from the urban area, so there is 
a great difference between the congestion problem of toll and the traffic jam problem of urban areas [4]. From the perspective of impact, the toll station congestion will affect the whole expressway section, and the impact range will be wider if it is serious; from the perspective of congestion time, the greater the mobility of vehicles, the greater the chain reaction of congestion will be.

\subsection{Objectives}

The toll management of expressways should be continuously improved in terms of system and form, especially to improve its degree of refinement. The goal of expressway toll refined management is to further reduce the error rate of toll business and improve customer satisfaction.

Under the guidance of refined management, the management of expressway toll station requires a more detailed division of the whole process of toll collection, and further clarify the basic responsibilities and rights of each staff in the toll station, so that everyone can better complete their own work under the guidance of refined management ideas. Moreover, through the refined management system and norms, we will guide, innovate and restrict the charging work of each employee, so that everyone can strictly abide by the refined management system and norms.

\section{Application of Data Analysis in Expressway Refined Management}

\subsection{Data Sources}

In order to realize the refined management of expressway, it is necessary to gather various types of data information and realize cross domain data fusion, including vehicle traffic data, telecommunication data, meteorological data, public security data, vehicle refueling data, parking data, etc. The data source mainly includes internal data of traffic management department and external shared data, as shown in Table 1.

Table 1 Data Sources For Expressway Refined Management.

\begin{tabular}{|l|l|l|}
\hline Data sources & Key data items & Usage \\
\hline Vehicle traffic data & $\begin{array}{l}\text { ETC customer data, ETC transaction data, MTC } \\
\text { transaction data, video image data, expressway } \\
\text { network data, toll station data, etc. }\end{array}$ & $\begin{array}{l}\text { Traffic flow monitoring; emergency } \\
\text { response; operation management support }\end{array}$ \\
\hline $\begin{array}{l}\text { Telecommunication } \\
\text { data }\end{array}$ & $\begin{array}{l}\text { User bill data, user online data, location service } \\
\text { data, etc. }\end{array}$ & $\begin{array}{l}\text { Real-time monitoring of road network } \\
\text { operation status; early warning information } \\
\text { release }\end{array}$ \\
\hline Public security data & $\begin{array}{l}\text { Motor vehicle registration data, driver's license } \\
\text { data, violation data }\end{array}$ & $\begin{array}{l}\text { Provide services for operation network } \\
\text { inspection }\end{array}$ \\
\hline $\begin{array}{l}\text { Meteorological } \\
\text { data }\end{array}$ & $\begin{array}{l}\text { Temperature, humidity, radiation index, PM2.5 } \\
\text { index, wind force, meteorological disaster, etc. }\end{array}$ & $\begin{array}{l}\text { Issue alert information; conduct road patrol; } \\
\text { implement traffic emergency response }\end{array}$ \\
\hline Parking data & $\begin{array}{l}\text { Parking data, customer data, parking payment } \\
\text { data, etc. }\end{array}$ & $\begin{array}{l}\text { Traffic congestion prediction; precision } \\
\text { marketing; expressway pricing }\end{array}$ \\
\hline
\end{tabular}

\subsection{Data Analysis Applications}

\subsubsection{Traffic Flow Trends Prediction}

Based on historical data of traffic distribution of vehicles on expressways at different time periods and different road sections, analyse the saturation of traffic flow, and provide policy support for vehicle current limit, dredging, and bypass scheme development through policy guidance, information display, and other methods.

(1) Expressway traffic volume analysis during holidays and special periods. Provides thematic analysis including traffic volume scale, congestion-prone sections, time period distribution, toll lanes in congested sections, lane traffic efficiency, expected end-of-congestion duration, noncongested sections, and detour recommendations, etc.

(2) Distribution trend analysis of road network traffic flow. Provides thematic analysis of various sections in the road network, average traffic volume, detour schemes and dredging strategies when roads are congested, etc.

(3) Traffic accident analysis. Provides thematic analysis of high-incidence periods and road 
sections, historical frequency, casualties, incidents and accident causes, and decision-making recommendations, etc.

(4) Traffic violation types and distribution analysis. Provides thematic analysis of frequent traffic violation time periods, road sections, causes, and accuracy of snapping points, etc.

(5) ETC lane construction decision analysis. Provide thematic analysis including ETC lane construction software, hardware input cost, service loss, service life, maximum load capacity, influencing factors, etc.

(6) Service area service quality analysis. Provides thematic analysis including service area traffic flow, average vehicle stay time, stay behaviour, consumer behaviour preferences, etc.

Through above thematic analysis of expressway operation data, it can give correct decisions to expressway management departments from a macro perspective and a strategic perspective, and realize the refined management of expressway ETC, thereby improving the overall operation level and profitability.

\subsubsection{Traffic Emergency Response Decision Support}

For sudden traffic accidents, natural disasters, and congestion caused by various reasons on the expressway, through data collection and analysis, it provides decision-making support for the advance formulation and prevention of traffic emergency treatment plans.

\subsubsection{Assistance in Auditing Traffic Evasion Fees}

The operation data of the transportation department show that, there are still many cases of fee stealing and evasion. It will indirectly increase the cost of road operation and management, extended the recovery period of road construction investment, and caused huge losses to the country [5]. Through the comprehensive analysis of public security data, monitoring data, etc., it can intelligently track the driving track of the target vehicle, automatically identify and record the vehicle traffic data, so that effectively help control vehicle evasion behaviour. The visual intelligent analysis system based on the application of high-speed expressway big data, such as monitoring, perception, research and early warning, is a necessary condition for vehicle audit and anti-evasion fee

\subsubsection{Refined Marketing Based on Customer Segmentation}

On the basis of multi-dimensional traffic data, establish user portrait and group segmentation, and then flexibly formulate various preferential policies to meet the personalized demands of different users and the needs of major users. The customer classification based on data mining can realize the precise marketing of expressway charging and reduce the operation cost of expressway.

\section{Countermeasures and Suggestions on Refined Management of Expressway Toll}

\subsection{Improve Big Data Application Environment}

There are administrative barriers between traffic management departments and agencies, and related data is scattered in different departments, and there is no mechanism for open interoperability and data sharing between departments. This has resulted in fragmentation and segmentation of data resources, which seriously affected the efficiency of traffic resource allocation. Therefore, there is an urgent need to completely break administrative barriers, achieve data access, and establish a unified standard system for data processing, application, and control. While opening up data, we should strengthen cooperation and communication among various traffic management departments, open up data barriers, and establish unified regulations, institutions, and mechanisms for management and application.

\subsection{Increase Financial Support for Technological Innovation}

The government should increase financial support for big data-related projects to encourage innovation by businesses and individuals, especially to develop related products, platforms or services based on traffic toll data. For the localized start-ups that are operating in favour of a 
expressway toll system or application, after comprehensively evaluating the externality of its effectiveness, the government should provide incentive subsidies and provide policy guidance for its development.

\subsection{Strengthen Privacy Protection}

Once big data applications are introduced into expressway toll management, the privacy and security of personal data of car owners will become particularly important. When applying for an ETC card, traffic department will collect the user's ID information, vehicle information, driving license information, etc. After the ETC card is activated, their recharge information, expressway driving track information, transaction information, and violation information will be collected. All these data belong to the owner's private information, but in order to provide users with personalized services better and more accurately, the openness, sharing, and fusion of data is necessary. Therefore, to solve the contradiction between data openness and privacy is an issue that expressway administration must be solved. For this reason, the state and the transportation department should issue the corresponding norms and standards, strictly control the privacy of citizens, and severely punish the enterprises or individuals who violate the relevant regulations.

\subsection{Improve Operation Management and Service Level}

The gradual implementation of the national network toll system has promoted the comprehensive upgrading and development of the expressway toll system. It not only improves the operation efficiency and traffic efficiency of toll system, but also improves the expressway toll management level. However, the expressway service, operation and charging mode still cannot keep up with the pace of intelligent transportation information construction. The toll system sets up a special information channel between expressway and motor vehicle, realizing the real-time interaction of human, vehicle and road information, and produces a lot of valuable information data. The expressway management department should make full use of these data, quickly and timely find and capture the problems in the operation of the road network and make quick decisions, so as to provide personalized services for car owners and optimize the road network structure.

\section{Conclusion}

Refined management is an important direction of expressway toll management. By improving the charging management rules and regulations and work requirements, we can make the charging management work more standardized, and can better promote the management level of toll stations.

Firstly, we elaborate main problems of expressway toll refined management, and put forward the objectives of expressway toll refined management. Then, we propose that data mining can be used to develop the application of expressway refined management. Finally, we put forward some countermeasures and suggestions on refined management of expressway toll, from the perspective of big data application environment, financial support, privacy protection and management and service level.

\section{Acknowledgements}

If any, should be placed before the references section without numbering.

\section{References}

[1] Ying, Jiangqian, Ando, Ryosuke. (2007). On the Effects of Central Japan Expressway's Commuter Toll Discount Policy in Nagoya Area. Journal of Tsinghua University Natural Science, vol.12, no. 2, pp. 151-157.

[2] Xing, J, Takahashi, H, Kameoka, H. (2010). Mitigation of Expressway Traffic Congestion Through Transportation Demand Management With Toll Discount. IET Intelligent Transport Systems, vol. 4, no. 1, pp. 50-59. 
[3] Pei Xucheng. (2017). Management and Application of Toll Collection Monitoring System of the Expressway. Modern industrial economy and informatization, vol. 007, no. 3, pp. 76-77, 85.

[4] S.-B. Li, W.-X. Dang, B.-B. Fu. (2015). Traffic Real-time Network States Estimation of Expressway Based on Toll Data. Jiaotong Yunshu Xitong Gongcheng Yu Xinxi/ Journal of Transportation Systems Engineering \& Information Technology, vol. 15, no. 4, pp. 63-69 and 84.

[5] Monica Singhania, Kamal Kumar. (2014). The Financial Viability of the YAMUNA Expressway. Emerging Markets Case Studies Collection, vol. 4, no. 4, pp. 1-20. 